\title{
Particle in Cell-Monte Carlo Collisions of a Plasma Column Driven by Surface Wave Plasma Discharges
}

\author{
Mohammad Sadegh Soltani Gishinia, Alireza Ganjovia, Majid Taraz ${ }^{\mathbf{b}}$, and Masood Saeed \\ aPhotonics Research Institute, Institute of Science and High Technology and Environmental \\ Sciences, Graduate University of Advanced Technology, Kerman, Iran \\ bFaculty of Physics, Shahid Bahonar University of Kerman, Kerman, Iran \\ corresponding author : Ganjovi@,kgut.ac.ir
}

Received: Aug. 28, 2016, Revised: Dec. 31, 2016, Accepted: Jan. 16, 2017, Available Online: Oct. 28, 2017

\begin{abstract}
In this work, applicability of Particle in Cell-Monte Carlo Collisions (PICMCC) simulation method for better understanding of the plasma physical mechanisms and real important aspects of a plasma column driven by surface wave plasma discharges that is used in plasma antennas is examined. Via the implementation of geometry and physical parameters of the plasma column to an Object Oriented PIC-MCC code, the plasma density, electrical conductivity, plasma kinetic energy and electric field inside the plasma column as its essential properties are obtained. The gas within the plasma column is taken to be argon which is kept at the low operational background pressures. The radial increasing and axial decreasing of the electric field in the plasma column is observed. Moreover, the plasma density reduces radially, while it is maximized along the axial positions. It is seen that, the density of charged particles and their corresponding current densities are maximized at the positions closer to the surface wave launcher.
\end{abstract}

KeYwORDS: PIC-MCC Simulation, Surface Wave Plasma Discharges, Plasma Antenna.

\section{I.INTRODUCTION}

Surface wave plasma discharges use the propagation of surface waves along the dielectric surface and plasma medium interface to produce and sustain the plasma discharge [1]. Considering of the nonlinear effects which are specific to the bounded plasma discharges, the appearance of the plasma surface solitary wave on the interface between the plasma column and the bounding dielectric medium was studied by
Stenflo [2]. On the basis of energy balance equations, Aliev [3] obtained the axial distribution of the produced electron density by the ionizing surface waves.

Initially, the surface wave produced plasmas were used in the plasma antennas by Borg et al. [4]. They showed that, the surface wave plasmas generate very low noises and can be used to produce plasma in the plasma antennas. The surface wave launcher (for example Surfatron [5]) surrounds only a small portion of the discharge vessel and antenna is fed from one end. Thus, the stealth capability of plasma antenna is improved owing to the reduction in the metal elements [6].

Sa et al. [7] used a self-consistent theory to simulate the low-pressure surface wave sustained gas discharges. Their theory had the capability of providing a complete description of the plasma density and surface wave field distribution in both the radial and the axial directions. The model was based on a complete set of equations including Maxwell, Boltzmann, continuity and momentum transfer equations with the boundary conditions for the surface wave field. They obtained the local collisional and transport data versus the surface wave electric field.

Kousaka et al. [8] studied the propagation of electromagnetic waves along the plasmadielectric interfaces in an azimuthally symmetric surface wave-excited plasma source. They used a 2D numerical analysis based on the 
finite difference time-domain (FDTD) approximation and, the Maxwell equations and non-magnetized cold plasma-electron equations were solved. They calculated different plasma electron densities that the electromagnetic waves can propagate as volume or surface waves.

A Finite-Element (FE) analysis of surface wave plasmas was used by Igarashi et al. [9]. They computed the resonant modes of the surface wave on the interface between the dielectric and plasma in a cylindrical vessel Moreover, Kabouzi et al. [10] examined the atmosphericpressure plasma columns sustained by surface waves using a self-consistent two-dimensional fluid-plasma model coupled with the Maxwell equations for argon discharge columns sustained at atmospheric pressure. Their simulation scheme was able to provide the full axial and radial structures of the surface-wave plasma column. Moreover, the distribution of electromagnetic fields for given discharge operating conditions were obtained.

Alves et al. [11] studied the surface-wave discharge columns in a cylindrical symmetry. They used a stationary, one-dimensional radial moment model including the continuity and the momentum transfer equations. They solved the electron mean energy transport equations selfconsistently coupled with the Poisson equation for the space-charge electrostatic field and the appropriated Maxwell equations for the surface wave electromagnetic field. In addition, Nikovski et al. [12] presented a 3D model of a surface-wave-sustained discharge at atmospheric pressures. While the Boltzmann equation was solved for plasma electrons, the behavior of plasma heavy particle was studied using the balance equations at a fixed axial profile. Furthermore, the model was included the dispersion relation of azimuthallysymmetric surface waves.

Despite of much research efforts on the understanding of the plasma physical mechanisms of the plasma columns sustained by surface wave discharges [7-12], study of the applicability of PIC-MCC simulation scheme to analyze its real important features could carry interesting results. Thus, in this paper, via implementation of the geometry and physical parameters of a surface wave sustained plasma column to an Object Oriented PIC-MCC (OOPIC) code, its plasma physical features through the essential properties of the plasma column, i.e., electric field in the plasma column, plasma and current densities, kinetic energy (or temperature) of the charged species and plasma column conductivity are studied. It will be shown that, the PIC-MCC simulation scheme is interestingly able to give further detailed perspectives about the plasma physical aspects of a surface wave sustained plasma column which could be helpful to perform more comprehensive experimental investigations.

\section{Simulation MethoD}

In this work, Object Oriented PIC-MCC (OOPIC) code is used to study the physical features of the plasma column of the plasma antenna driven by a surface wave through its essential properties, i.e., electric field, plasma density, conductivity and kinetic energy of charged species inside the plasma column. The considered discharge mechanism for the plasma column of a plasma antenna is the surface wave discharge getting excited by a common RF generator at low pressure background gases [5, 13-15]. The surface wave motion on the dielectric tube and gaseous media of the plasma column interface results in the formation of the striations inside the plasma column [16].

The Particle in Cell-Monte Carlo Collisions (PIC-MCC) simulation scheme as a kinetic method is able to estimate the basic features of the low pressure gaseous discharge media e. g., surface wave sustained plasma columns of the plasma antennas that cannot be easily simulated with the other simulation methods such as fluid models [17-21]. These features are mostly including the nonlocal plasma kinetic effects, ohmic heating, and striation formation inside these plasma columns [22, 23, 24]. On the other hand, the differential cross sections for collisions, that are essential in the plasma column, can be easily implemented in PICMCC simulations. This method provides the evolution of different features of surface wave 
sustained plasma columns of plasma antenna as it works in RF range. While the PIC-MCC simulation method can overcome many limitations of the other methods, it is computationally expensive and typically limited to simple geometries with few gas species such as plasma columns that are used in plasma antennas. However, the obtained results can also be used to predict the spatial behavior and physical properties of the surface wave sustained plasma columns that are used in plasma antennas to propose the novel designs for the performance improvements $[25,26]$.

The PIC-MCC simulation method takes the advantage of collective behaviors in the plasma discharge media. This modeling scheme is based on the modeling of reduced number of computer particles (or super-particles) [17-20]. Generally, one super-particle per 105 to 107 real particles is chosen in the existing reported literatures. In spite of this much of reduction in the number of particles, Birdsall [20] showed that, the plasma physics is retained by this method for plasma simulation. The trajectory of these super-particles is tracked via solving of the fundamental equations, i.e., NewtonLorentz coupled with Maxwell equations. Moreover, no assumption has been made on their velocity distribution. Thus, the kinetic behavior of each species is modeled through a very little approximation. In this method, the simulations are performed for electrons and ions of plasma and, background neutrals are assumed to have a uniform spatial distribution.

However, in the PIC-MCC simulation method, a knowledge of relevant collision cross sections (or transport parameters) in the discharge and the boundary conditions such as the applied voltage and discharge current is necessary. In low-pressure discharges, such as plasma columns that are used in plasma antennas, the particle distribution functions may deviate from thermal equilibrium. Furthermore, the so-called local field approximation may not hold. Then, it is advisable to go for kinetic and microscopic simulation schemes rather than the fluid and macroscopic descriptions [26].
Generally, in the new generation of plasma antennas, the excitation of gaseous medium inside the plasma antenna tube and plasma formation is based on the traveling of the surface waves along the interface of dielectric (glass) tube and gaseous medium of antenna $[13,27]$. The surface wave electric field results in the moving of ionization front and the discharge plasma build-up will fill the entire of glass tube. The plasma column within the plasma antenna is considered in the cylindrical coordinates, as shown in Fig. 1. In this coordinates system, the possible propagating modes of surface waves are defined by their field intensity dependence upon the azimuthal angle $\varphi$. This field intensity in the case of uniform media varies as $\exp (\operatorname{im} \varphi)$ for traveling waves, where $m$ is an integer defining the mode of propagation [27]. The $m=0$ mode for surface wave produced plasma is considered and there is an azimuthal symmetry in the plasma column. Thus, the axisymmetric coordinates, $(z, r)$, will be sufficient to describe the problem geometry. In this cylindrical geometry, the $z$ axis is along dielectric tube axis and r-axis is along its radius. The origin of coordinate system is placed at one end of the tube and at the center of the surface wave launcher. The surface wave launcher, i.e., Surfatron [5], is mounted at one end $(\mathrm{z}=0)$ of the tube and excites the traveling surface waves to produce and sustain the plasma column discharge inside the plasma antenna [13-15, 27].

In this work, Object Oriented PIC-MCC (OOPIC) code is used to study the physical features of the plasma column of the plasma antenna driven by a surface wave through its essential properties, i.e., electric field, plasma density, conductivity and kinetic energy of charged species inside the plasma column. The considered discharge mechanism for the plasma column of a plasma antenna is the surface wave discharge getting excited by a common RF generator at low pressure background gases [5, 13-15]. The surface wave motion on the dielectric tube and gaseous media of the plasma column interface results in the formation of the striations inside the plasma column [16]. 
The Particle in Cell-Monte Carlo Collisions (PIC-MCC) simulation scheme as a kinetic method is able to estimate the basic features of the low pressure gaseous discharge media e. g., surface wave sustained plasma columns of the plasma antennas that cannot be easily simulated with the other simulation methods such as fluid models [17-21]. These features are mostly including the nonlocal plasma kinetic effects, ohmic heating, and striation formation inside these plasma columns $[22,23,24]$. On the other hand, the differential cross sections for collisions, that are essential in the plasma column, can be easily implemented in PICMCC simulations. This method provides the evolution of different features of surface wave sustained plasma columns of plasma antenna as it works in RF range. While the PIC-MCC simulation method can overcome many limitations of the other methods, it is computationally expensive and typically limited to simple geometries with few gas species such as plasma columns that are used in plasma antennas. However, the obtained results can also be used to predict the spatial behavior and physical properties of the surface wave sustained plasma columns that are used in plasma antennas to propose the novel designs for the performance improvements $[25,26]$.

The PIC-MCC simulation method takes the advantage of collective behaviors in the plasma discharge media. This modeling scheme is based on the modeling of reduced number of computer particles (or super-particles) [17-20]. Generally, one super-particle per 105 to 107 real particles is chosen in the existing reported literatures. In spite of this much of reduction in the number of particles, Birdsall [20] showed that, the plasma physics is retained by this method for plasma simulation. The trajectory of these super-particles is tracked via solving of the fundamental equations, i.e., NewtonLorentz coupled with Maxwell equations. Moreover, no assumption has been made on their velocity distribution. Thus, the kinetic behavior of each species is modeled through a very little approximation. In this method, the simulations are performed for electrons and ions of plasma and, background neutrals are assumed to have a uniform spatial distribution.
However, in the PIC-MCC simulation method, a knowledge of relevant collision cross sections (or transport parameters) in the discharge and the boundary conditions such as the applied voltage and discharge current is necessary. In low-pressure discharges, such as plasma columns that are used in plasma antennas, the particle distribution functions may deviate from thermal equilibrium. Furthermore, the so-called local field approximation may not hold. Then, it is advisable to go for kinetic and microscopic simulation schemes rather than the fluid and macroscopic descriptions [26].

Generally, in the new generation of plasma antennas, the excitation of gaseous medium inside the plasma antenna tube and plasma formation is based on the traveling of the surface waves along the interface of dielectric (glass) tube and gaseous medium of antenna $[13,27]$. The surface wave electric field results in the moving of ionization front and the discharge plasma build-up will fill the entire of glass tube. The plasma column within the plasma antenna is considered in the cylindrical coordinates, as shown in Fig. 1. In this coordinates system, the possible propagating modes of surface waves are defined by their field intensity dependence upon the azimuthal angle $\varphi$. This field intensity in the case of uniform media varies as $\exp (\operatorname{im} \varphi)$ for traveling waves, where $\mathrm{m}$ is an integer defining the mode of propagation [27]. The $\mathrm{m}=0$ mode for surface wave produced plasma is considered and there is an azimuthal symmetry in the plasma column. Thus, the axisymmetric coordinates, $(z, r)$, will be sufficient to describe the problem geometry. In this cylindrical geometry, the zaxis is along dielectric tube axis and r-axis is along its radius. The origin of coordinate system is placed at one end of the tube and at the center of the surface wave launcher. The surface wave launcher, i.e., Surfatron [5], is mounted at one end $(z=0)$ of the tube and excites the traveling surface waves to produce and sustain the plasma column discharge inside the plasma antenna $[13-15,27]$.

In this work, plasma antenna is considered as a plasma column that is excited by an electromagnetic surface wave [6]. As shown in 
Fig. 1, the plasma antenna is a dielectric tube that is evacuated by conventional rotary or diffusion pumps and filled by argon gas at low background gas pressures (0.01-0.1Torr). The power generator works in RF range (100$500 \mathrm{MHz}$ ) with the power of up to $100 \mathrm{~W}$. The required power for initial breakdown in the antenna tube is about 10 to $20 \mathrm{~W}$ at a gas pressure of 0.015Torr [6]. As shown in Fig. 1, a matching network is used to transmit all the RF power from the generator to the plasma discharge medium by matching the impedances.

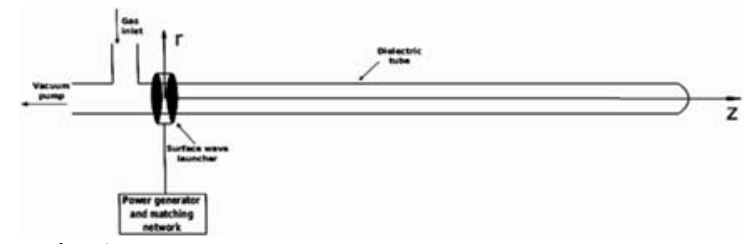

Fig. 1. Schematic diagram of plasma antenna and the simulation coordinates.

In the structure of the plasma antenna, the surface wave electric field accelerates the plasma electrons. The accelerated electrons dissipate their energy through elastic and inelastic collisions with neutral atoms inside the plasma column. Owing to the nature of surface wave produced plasmas, ionization of second and higher order argon atoms are ignorable and, only the two charged species (electrons and argon ions $(\mathrm{Ar}+))$ are taken into the account [27]. The surface wave gets excited from one end of plasma column $(\mathrm{z}=0)$ by the surface wave launcher and damps as it propagates along the internal surface of dielectric tube. This wave transfers some part of its energy to the plasma at each point on the dielectric tube surface. Moreover, in obtaining the boundary conditions for plasma column in the PIC-MCC simulation scheme, the exponential damping of the electromagnetic wave along the sidelong surface of the tube is assumed [13-16,27].

In the performed simulations, it is assumed that the surface wave power will be damped from $z=0$ in the axial direction, since its power goes for ionization of the antenna gaseous medium. The applied boundary conditions for electric potential and plasma density are listed in Table 1.
Table 1 Applied boundary conditions on the PICMCC model for plasma column

\begin{tabular}{cll}
\hline \hline Boundary & $\begin{array}{l}\text { Boundary } \\
\text { Conditions for } \\
\text { Potential }\end{array}$ & $\begin{array}{l}\text { Boundary } \\
\text { Conditions for } \\
\text { Plasma Density }\end{array}$ \\
\hline \hline $\mathrm{r}=\mathrm{r}_{0}, 0 \leq \mathrm{z} \leq \mathrm{z}_{0}$ & $\begin{array}{l}\text { Dirichlet } \\
\left(\Phi=\Phi_{0} \sin \omega \mathrm{t}\right)\end{array}$ & $\begin{array}{l}\text { Neumann } \\
(\partial \mathrm{n} / \partial \mathrm{r}=\text { constant })\end{array}$ \\
$0 \leq \mathrm{r} \leq \mathrm{r}_{0}, \mathrm{z}=0$ & $\begin{array}{l}\text { Dirichlet } \\
\left(\Phi=\Phi_{0} \sin \omega \mathrm{t}\right)\end{array}$ & $\begin{array}{l}\text { Neumann } \\
(\partial \mathrm{n} / \partial \mathrm{z}=\text { constant })\end{array}$ \\
$0 \leq \mathrm{r} \leq \mathrm{r}_{0}, \mathrm{z}=\mathrm{z}_{0}{ }^{*}$ & Dirichlet $(\Phi=0)$ & $\begin{array}{l}\text { Neumann } \\
(\partial \mathrm{n} / \partial \mathrm{r}=\text { constant })\end{array}$ \\
\hline The values of $\mathrm{r}_{0}$ and $\mathrm{z}_{0}$ are given in Table 2.
\end{tabular}

In the PIC-MCC simulation scheme, particles are defined in a continuum position and velocity space. Field values are defined at discrete locations in space. Particle and field values are advanced sequentially in time, starting from the initial conditions. The particle equations of motion are solved at every time step, using field values interpolated from the discrete grid to particle locations. The force on every particle is computed by interpolation of the field values from the grid position to the given particle positions. The position and velocities of each particle is next updated based on the solution of the classical equation of motion. Next, particle boundary conditions are applied [13]. For modeling of collisions, the Monte Carlo Collision (MCC) scheme is applied [14]. Source terms for the field equations are accumulated from the particle locations to the grid locations. Then, the field values are advanced by one time-step, and the time loop starts again (Fig. 2) [17-20].

In the PIC-MCC scheme, each super-particle represents one or much larger number of real particles and is designated in continuum space by its position and velocity. Initially, a Maxwellian distribution is assumed for electrons and ions. Further, the particles are uniformly distributed inside the plasma column. Particle boundary conditions such as absorption and emission are used to account for the relation between the plasma discharge current in the plasma tube and the current in the external circuit. When an electron or ion passes from the discharge into an end wall, it adds to the wall charge and is deleted from the list of active particles. Additionally, secondary emissions occur when a charged particle 
impacts the wall of the tube and causes the ejection of electrons from their surfaces [19].

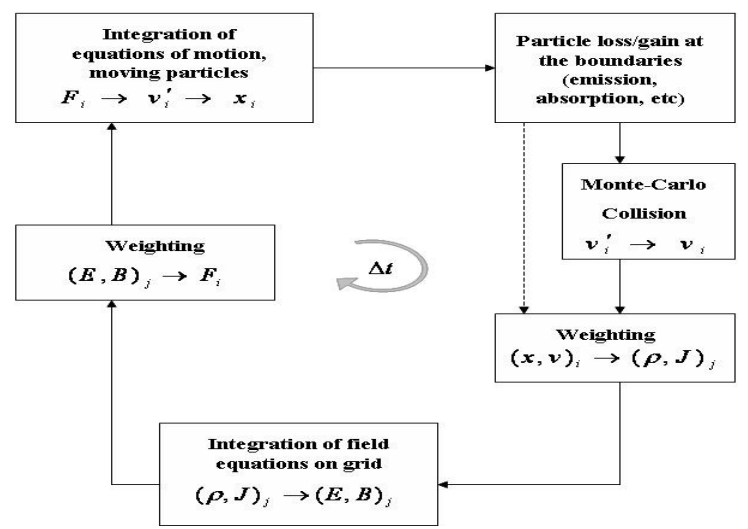

Fig. 2. Schematic representation of the computation sequence for PIC-MCC method [17].

The potentials and fields inside the plasma column are obtained using Poisson's equation:

$\nabla \cdot \varepsilon_{0} \nabla \Phi(\mathbf{r}, t)=\rho(\mathbf{r}, t)$

where $\rho$ is the net charge density which is zero at the charge neutrality condition. The potential (Ф) can be separated into Poisson and Laplace parts $[17,21]$ :

$\Phi=\Phi_{P}+\sum_{\text {Boundaries }} \Phi_{L}$

where $\Phi_{P}$ and $\Phi_{L}$ represent the Poisson and Laplacian parts of electrical potential $(\Phi)$. Therefore, the field may be described by the combination of the following equations:

$$
\begin{aligned}
& \nabla \cdot \varepsilon_{0} \nabla \Phi_{P}=-\rho \\
& \nabla \cdot \varepsilon_{0} \nabla \Phi_{L i}=0
\end{aligned}
$$

The boundary condition for equation (3) is $\Phi_{P}=0$ on all boundaries as the Poisson field is solely due to space charge in the medium. For each boundary with a Dirichlet condition, equation (4) is solved for $\Phi_{i}=0$ on the equipotential surface, and $\Phi=0$ elsewhere which gives us $\Phi_{L i}$ (the potential due to the $i^{\text {th }}$ Laplacian field). $\Phi_{L}$ is obtained by the superposition of all the Laplacian fields. This method neglects charges induced by a driven electrode on other boundaries which are connected to an external circuit. However, solving of the field Poisson equation with boundaries and circuits is possible [19].
In this work, an X11- based Object Oriented Particle-In-Cell (XOOPIC) code is used [28]. This code is an electromagnetic program and solves Maxwell's equations on a mesh to selfconsistently advance the particles in time and space [28]. The code is developed using the PIC-MCC simulation scheme and is written in $\mathrm{C}++$ programing language [29]. This $\mathrm{C}++$ program is designed to simulate the spatiotemporal behaviour of all the charged particles in a $2 \mathrm{D}$ cylindrical geometry. This code was developed by Plasma Theory and Simulation Group (PTSG) [30] to apply the object-oriented programing technique in the kinetic simulations of plasma discharges. The object-oriented paradigm advances the PIC-MCC modelling and increases flexibility and extensibility [28, 29].

For stability and accuracy of calculations, the Courant-Levy stability criterion on the time step is given by [17]:

$$
\Delta t \leq \frac{1}{c \sqrt{(\Delta z)^{-2}+(\Delta r)^{-2}}}
$$

Thus, choosing the simulation parameters such as time step $(\Delta t)$ and cell sizes $(\Delta z$ and $\Delta r)$ in the cylindrical coordinates has an important role in the high accuracy and stability of simulation [31]. The velocity and energy of charged particles should be considered in choosing of the simulation time steps and, it has to be lower than the required time for a particle topass through a cell. The time step $(\Delta t)$ is inversely proportional to the plasma frequency. Usually, there must be $\omega_{p e .} \Delta t \leq 1.62$ [19], where, $\omega_{p e}$ is the plasma frequency and, generally, is defined as follows [32]:

$\omega_{p e}=\left(\frac{4 \pi n_{e}}{m_{e}}\right)^{1 / 2}$

where, $n_{e}$ is the electron density and $m_{e}$ is the electron mass. On the other hand, the mesh spacing in each direction, $h$, should be a fraction, $f$, of the Debye length, i.e., 


$$
h=f \lambda_{D}=f\left(\frac{\varepsilon_{0} T_{e}}{e n_{e}}\right)^{1 / 2} \approx 7000 f\left(\frac{T_{e}}{n_{e}}\right)^{1 / 2}
$$

where, $T_{e}(\mathrm{eV})$ is the electron temperature, and $f$ is usually chosen to be about 0.5 [19]. Generally, in the common plasma columns operating based on surface wave discharges, the electron temperature (or the kinetic energy of electrons) is in the order of 1-5 eV [14].

The magnitude of plasma current density can be obtained as follows:

$J=\sum_{j} e_{j} V_{d j}$

where $V_{d j}=\sqrt{V_{r j}^{2}+V_{\varphi j}^{2}+V_{z j}^{2}}$ is the drift velocity of $j^{\text {th }}$ particle and $e_{j}$ and electric charge of $j^{\text {th }}$ particle and summation is over all the plasma particles (electrons for electronic current and ions for ionic current). Moreover, the kinetic energy of a charged particle (electron or ion) in a plasma column is obtained using $K_{j}=1 / 2 m_{j} V_{d j}^{2}$, where $m_{j}$ is mass of charged particle (electron or ion) [32].

Table 2. Simulation Parameters

\begin{tabular}{ll}
\hline \hline Parameter & Value \\
\hline \hline $\begin{array}{l}\text { Number of cells in length }(\mathrm{z}) \\
\text { direction }\end{array}$ & 120 \\
$\begin{array}{l}\text { Number of cells in radius }(\mathrm{r}) \\
\text { direction }\end{array}$ & 5 \\
$\begin{array}{l}\text { Number of simulation cycles } \\
\text { Time step }(\Delta t)\end{array}$ & $10^{7}$ \\
Plasma column length $\left(z_{0}\right)$ & $10^{-11} \mathrm{~s}$ \\
Plasma column radius $\left(r_{0}\right)$ & $0.3 \mathrm{~m}$ \\
Number of physical particles to & $0.015 \mathrm{~m}$ \\
computer particles (np2c) & $10^{7}$ \\
Pressure & $0.015 \mathrm{Torr}$ \\
Gas & Ar (Argon) \\
RF generator frequency & $200 \mathrm{MHz}$ \\
Geometry & 0 (Cylindrical) \\
Dielectric tube relative & 5 for glass \\
permittivity & \\
\hline
\end{tabular}

\section{III.RESULTS AND DISCUSSION}

In this section, the general results on the essential properties of the plasma column of the plasma antenna using a two dimensional PICMCC simulation scheme are presented. These properties are mainly including the electric field, plasma and current densities and kinetic energy of electrons. The presented boundary conditions in Table I are imposed on the PICMCC simulation model. The simulations are performed with the chosen operational parameters presented in table II. It must be noted that, the numerical simulations are carried out using a desktop computer with 2472 hours runs.

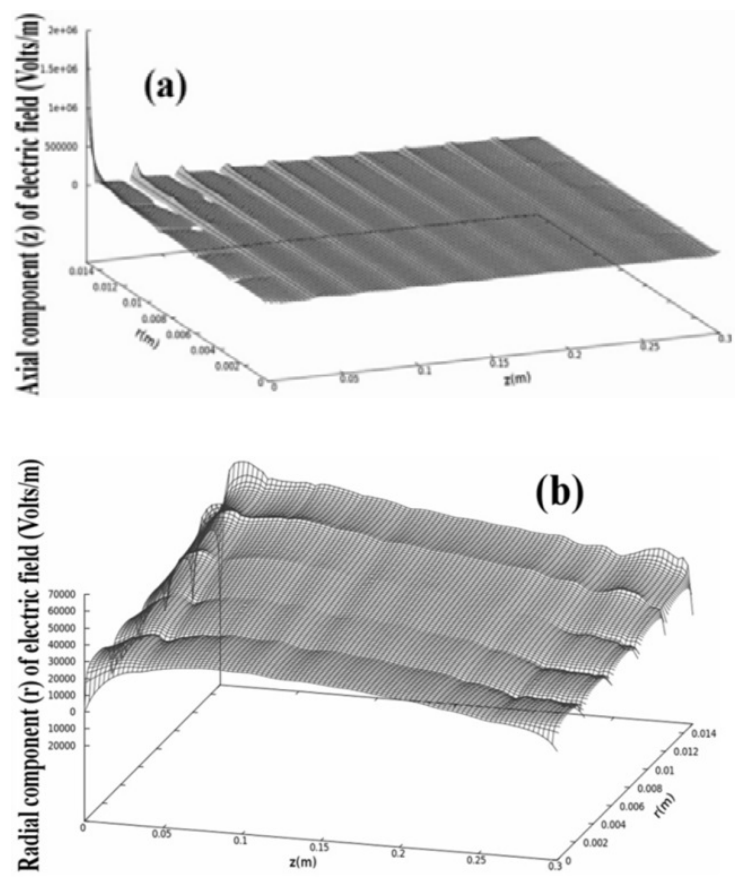

Fig. 3. Axial and radial variations of components of electric field (a) $z$-component $\left(E_{z}(r, z)\right)$, (b) $r$ component $\left(E_{r}(r, z)\right)$.

Figure 3 shows the spatial variations of the electric field components $\left(E_{z}, E_{r}\right)$ inside the plasma column. Due to the exponential dissipation of surface wave energy [13-15, 27] and formation of plasma along the tube axis (zaxis), the electric field damps exponentially along the tube axis. Moreover, the components of electric field increase radially and get maximized on the dielectric tube surface $(r=0.015 \mathrm{~m})$. Moreover, to show the direction of electric field at each point inside the plasma column, an arrow plot for electric field vector in the plasma column is shown in Fig. 4. The size of arrows is directly related to the strength of the electric field at the point located inside the plasma column. The direction of arrows is pointing corresponds to the orientation of the 
vector electric field at the particular point in the plasma column. The experimental and numerical studies by Kumar and Borg [16] on the plasma antennas and Moisan et al. [14] on the surface wave plasma columns show the importance of the electric field calculations. Kumar and Borg [16] showed experimentally that, the electric field exponentially decreases along the axis of plasma column and, increases radially. They concluded that, the axial variation of electric field is an indication of the power attenuation along the length of the plasma column [16].

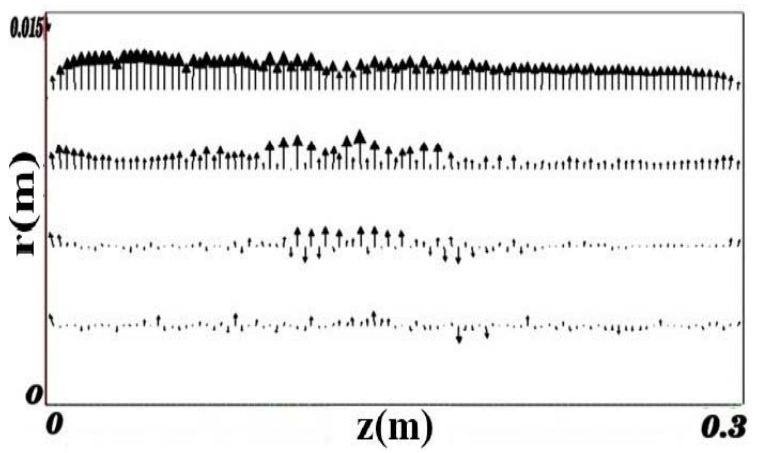

Fig. 4. Arrow plot presentation of the total electric field in $(r, z)$ plane.

The spatial variations of the plasma density inside the plasma column are shown in Fig. 5. As shown, increasing of the column radius results in the reduction of the plasma density. The plasma density is higher at the center of the plasma column and falls radially. This finding is in good agreement with the obtained experimental results by Kumar et al. [33] and Moisan et al. $[14,15]$. Moreover, the density of electrons along the plasma column (z-axis) for two different radial positions (i.e., $r=0.003 \mathrm{~m}$ and $\mathrm{r}=0.006 \mathrm{~m}$ ) is shown in the Fig. 5(b).As seen, the electron density is maximized. At lower axial positions from surface wave launcher, due to the lower effects of space charges field, the electron density reduces. On the other hand, owing to the reduction in the ionization strength of the surface wave at higher axial positions, the electron density, again, reduces. The axial non-uniformity (i.e., $\mathrm{dn}(\mathrm{z}) / \mathrm{dz} \leq 0)$ of the plasma discharge at the end of plasma tube is an intrinsic feature of plasma columns that are sustained by the surface waves. This is in agreement with the performed experiments by Moisan et al. [14].
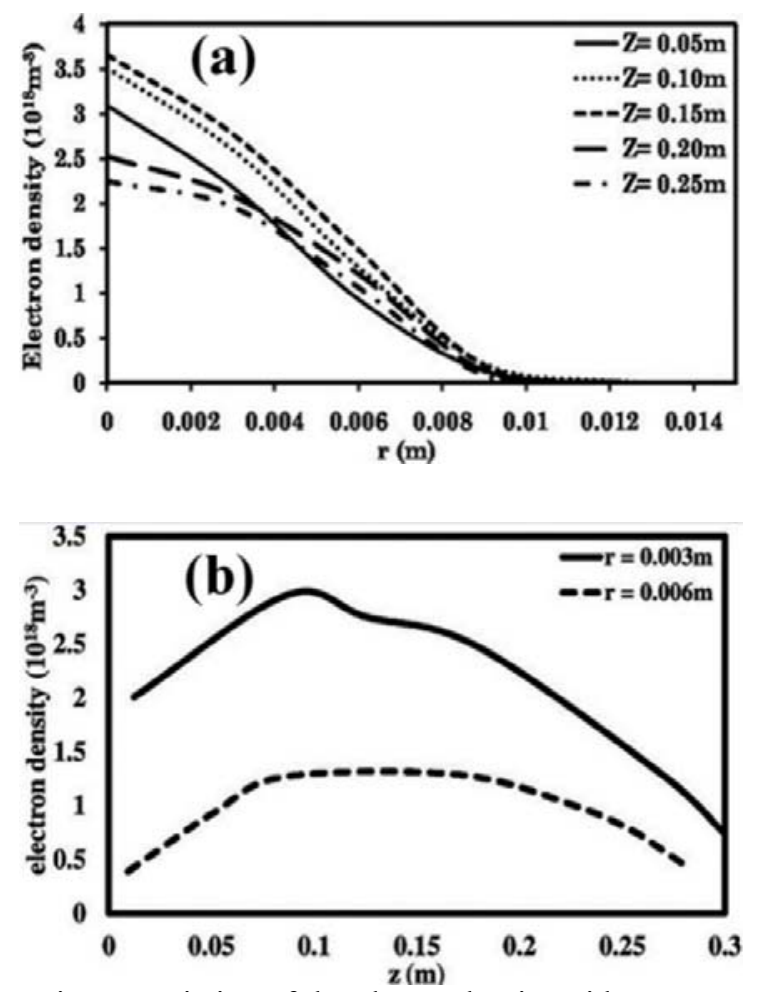

Fig. 5. Variation of the plasma density with respect to (a) the radius of plasma column at different axial positions i.e., $0.05,0.10,0.15,0.20,0.25 \mathrm{~m}$ from surface wave launcher,(b) the axial positions at two different radial positions, i.e., $r=0.003 \mathrm{~m}$ (solid line) and $\mathrm{r}=0.006 \mathrm{~m}$ (dashed line).

In summary, at higher radial positions, the electric field is higher, but the plasma density is interestingly lower. Based on Poisson equation (equation 1), this effect is owing to the fact that, the electric field in a surface wave plasma column has two origins, i.e., the traveling surface wave field and the space charges electric field. Near the dielectric tube surface, the plasma electrons experience a higher electric field from the surface wave electric field. Thus, they gain more kinetic energy to cause the collisional ionization process inside the plasma column and, the electric field forces electrons to move and ionize argon atoms near the tube axis [34].

It is well known that, the distribution of the plasma discharge current in the plasma column plays an important role in the radiation characteristics of the plasma column [6]. The 
PIC-MCC simulation method has the capability of calculation of the plasma current density in the plasma column. Figure 6 shows the electronic and ionic components of the discharge current density of the plasma column which behaves similar to the plasma density. As can be seen, both the electronic and ionic current densities are maximized at about 0.05 $0.07 \mathrm{~m}$ and, monotonically decrease at higher axial positions. Such results are obtained in the performed experiments by Kumar and Bora [6]. Additionally, Kumar and Bora [6] fitted an empirical equation on the obtained experimental data for the distribution of plasma current, I(z). To prove the capability of the PICMCC simulation method to study the plasma physical features of plasma column and making a reasonable comparison with the existing experimental results by Kumar and Bora, a simple parametric fit is performed on the axial variations of plasma current density magnitude at the axis of plasma column $(\mathrm{r}=0)$. This curve fitting has a shifted Gaussian shape as follows:

$$
j(z)=j(0) \exp \left\{-\frac{(z-a)^{2}}{2 b^{2}}\right\}
$$

where $j(0)$ is the magnitude of the current density at $z=0$ and the constants $a$ and $b$ are 0.111 and 0.131 for electronic current density and 0.107 and 0.104 for ionic current density, respectively. Moreover, owing to the higher ions mass and its direct effect on their velocity, the electronic current density is about $10^{4}$ times higher than that for the plasma ions.

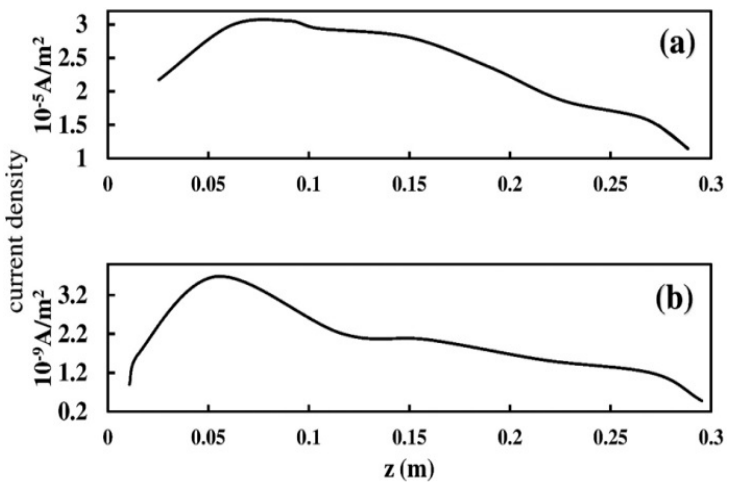

Fig. 6. The axial variations of the current density at the center of the plasma column $(\mathrm{r}=0)$, (a) electrons and (b) ions.
The axial variations of the kinetic energy (or temperature) of electrons and ions within plasma column are shown in Fig. 7. As seen, the kinetic energy of charged species versus axial positions (z) peaks at the same positions as the plasma current density. However, again, due to much higher mass of ions and its influence on their velocity, the kinetic energy of electrons is very much higher than that for ions.

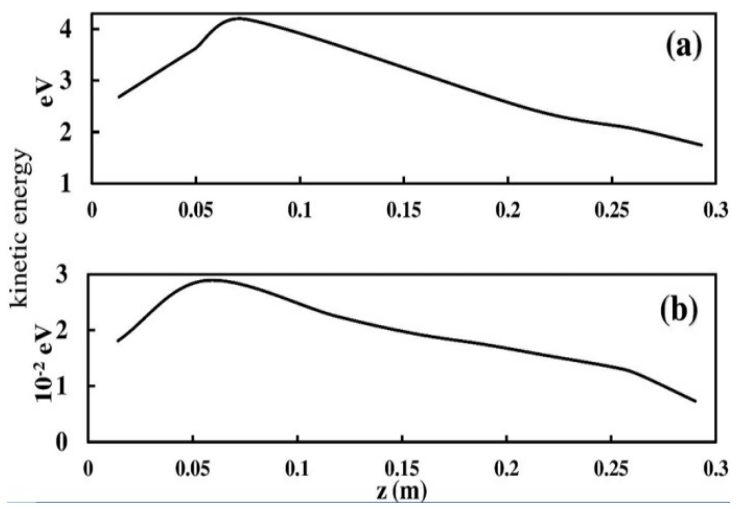

Fig. 7 The axial variations of the kinetic energy at $\mathrm{r}=0$, (a) electrons and (b) argon ions.

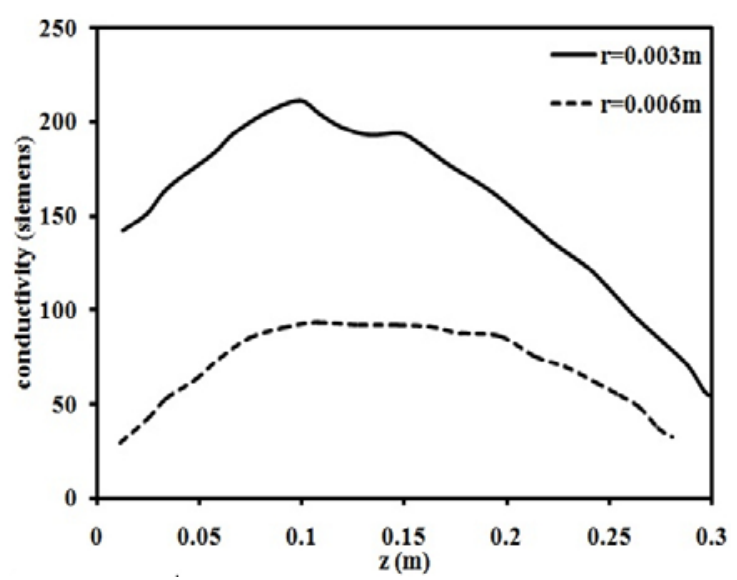

Fig. 8. Variation of the conductivity with respect to the axial positions at two different radial positions, i.e., $r=0.003 \mathrm{~m}$ (solid line) and $r=0.006 \mathrm{~m}$ (dashed line).

The plasma column electrical conductivity of the plasma antenna column is one of its interesting features which can be estimated using the PIC-MCC simulation scheme. As seen in Fig. 8, the axial variations of the electrical conductivity of the plasma column for two different radial positions (i.e., $r=0.003 \mathrm{~m}$ and $r=0.006 \mathrm{~m})$ is maximized at about $\mathrm{z}=10 \mathrm{~cm}$. The observed trend in the electrical 
conductivity is in agreement with the experimental findings by Kumar and Bora [6].

\section{IV.CONCLUSION}

In this work, a two dimensional particle in cellMonte Carlo collisions (2D-PIC-MCC) simulation model was found to be suitable to study the physical mechanisms and effective features of the plasma column of a plasma antenna driven by surface wave plasma discharges through its essential properties. The plasma antenna column geometry and its physical parameters were implemented to an Object Oriented PIC-MCC code. The axial damping and radial increasing of the electric field was observed. Moreover, the charged particles density inside the plasma column decreases both radially and axially. The higher electric field and lower plasma density at higher axial positions was explained based on the traveling surface wave electric field and the spatial charges field. The maximum values of plasma density, its current density and kinetic energy of charged particles were seen in the proximity of the surface wave launcher.

\section{ACKNOWLEDGMENT}

We would like to acknowledge the Institute of Science and High Technology and Environmental Sciences for financial support within the project no. 7.2988-16/6/1393.

\section{REFERENCES}

[1] Y.A. Akimov and K. Ostrikov, "Interaction of transverse electromagnetic waves with counterpropagating surface waves at a plasmadielectric interface," Phys. Scr., vol. 76, pp. 461-465, 2007.

[2] L. Stenflo, "Theory of nonlinear plasma surface waves," Phys. Scr., vol. 1996, pp. 59-62, 1996.

[3] Y.M. Aliev, A.V. Maximov, H. Schlüter, and A. Shivarova, "On the axial structure of surface wave sustained discharges," Phys. Scr., vol. 51, pp. 257-262, 1995.

[4] G.G. Borg, J.H. Harris, N.M. Martin, D. Thorncraft, R. Milliken, D.G. Miljak, B. Kwan, $\mathrm{T}$. Ng, and J. Kircher, "Plasmas as antennas: Theory, experiment and applications," Phys. Plasmas, vol. 7 pp. 2198-2202, 2000.
[5] M. Moisan, Z. Zakrzewskit, and R. Pantel, "The theory and characteristics of an efficient surface wave launcher (surfatron) producing long plasma columns," J. Phys. D: Appl. Phys., vol. 12, pp. 219-238, 1979.

[6] R. Kumar and D. Bora, "A reconfigurable plasma antenna," J. Appl. Phys., vol. 107, p. 053303, 2010.

[7] A.B. Sá, C.M. Ferreira, S. Pasquiers, C. BoisseLaporte, P. Leprince, and J. Marec, "Selfconsistent modeling of surface wave produced discharges at low pressures," J. Appl. Phys., vol. 70, pp. 4147-4158, 1991.

[8] H. Kousaka and K. Ono "Numerical Analysis of the Electromagnetic Fields in a Microwave Plasma Source Excited by Azimuthally Symmetric Surface Waves," Jpn. J. Appl. Phys., vol. 41, pp. 2199-2206, 2002.

[9] H. Igarashi, K. Watanabe, T. Ito, T. Fukuda, and T. Honma, "A finite-element analysis of surface wave plasmas," IEEE Trans. Magn., vol. 40, pp. 605-608, 2004.

[10] Y. Kabouzi, D.B. Graves, E. CastañosMartínez, and M. Moisan, "Modeling of atmospheric-pressure plasma columns sustained by surface waves," Phys. Rev. E, vol. 75, pp. 016402, 2007.

[11] L.L. Alves, S. Letout, and C. Boisse-Laporte, "Modeling of surface-wave discharges with cylindrical symmetry," Phys. Rev. E, vol. 79, pp. 016403 (1-18), 2009.

[12] M. Nikovski, Zh. Kissovski, and E. Tatarova, "Model of a surface-wave discharge at atmospheric pressure with a fixed profile of the gas temperature," J. Phys.: Conf. Ser., vol. 700, pp. 012014, 2016.

[13]M. Moisan, A. Shivarova, and A.W. Trivelpiece, "Experimental investigations of the propagation of surface waves along a plasma column," Plasma Phys., vol. 24, pp. 1331-1400, 1982.

[14] M. Moisan, M. Ferreira, Y. Hajlaoui, D. Henry, J. Hubert, R. Pantel, A. Ricard, and Z. Zakrzewski, "Properties and applications of surface wave produced plasmas," Revue. Phys. Appl., vol. 17, pp. 707-727, 1982.

[15]M. Moisan and Z. Zakrzewski, "Plasma sources based on the propagation of electromagnetic surface waves," J. Phys. D: Appl. Phys., vol. 24, pp. 1025-1048, 1991. 
[16]R. Kumar and D. Bora, "Experimental investigation of different structures of a radio frequency produced plasma column,"Phys. Plasmas, vol. 17, pp. 043503 (1-7), 2010.

[17]J.P. Verboncoeur, "Particle simulation of plasmas: review and advances," Plasma Phys. Control. Fusion, vol. 47, pp. A231-A260, 2005.

[18] V. Vahedi and M. Surendra, "A Monte Carlo collision model for the particle-in-cell method: applications to argon and oxygen discharges," Comput. Phys. Commun., vol. 87, pp. 179-198, 1995.

[19]C.K. Birdsall and A.B. Langdon, Plasma Physics Via Computer Simulation, New York: McGraw-Hill, 1985.

[20] C.K. Birdsall, "Particle-in-cell charged-particle simulations, plus Monte Carlo collisions with neutral atoms, PIC-MCC," IEEE Trans. Plasma Sci., vol. 19, pp. 65-85, 1991.

[21] V. Vahedi and G. DiPeso, "Simultaneous Potential and Circuit Solution for TwoDimensional Bounded Plasma Simulation Codes," J. Comput. Phys. vol. 131, pp. 149163, 1997.

[22] G.Y. Park, S.J. You, F. Iza, and J.K. Lee, "Abnormal Heating of Low-Energy Electrons in Low-Pressure Capacitively Coupled Discharges," Phys. Rev. Lett., vol. 98 pp. 085003, 2007.

[23]H.C. Kim and J.K. Lee, "Mode Transition Induced by Low-Frequency Current in DualFrequency Capacitive Discharges," Phys. Rev. Lett., vol. 93, pp. 085003 (1-4), 2004.

[24] V.I. Kolobov, "Striations in rare gas plasmas," J. Phys. D: Appl. Phys., vol. 39, pp. R487R506, 2006.

[25] Y.J. Hong, M. Yoon, F. Iza, G.C. Kim, and J.K. Lee, "Comparison of fluid and particle-in-cell simulations on atmospheric pressure helium microdischarges," J. Phys. D: Appl. Phys., vol. 41, pp. 245208 (1-5), 2008.

[26] H.C. Kim, F. Iza, S.S. Yang, M. RadmilovicRadjenovic, and J.K. Lee, "Particle and fluid simulations of low-temperature plasma discharges: benchmarks and kinetic effects," J. Phys. D: Appl. Phys., vol. 38, pp. R283-R301, 2005.

[27] O.A. Popov, High Density Plasma Sources Design, Physics and Performance, Massachusetts: Elsevier, 1996.
[28] J.P. Verboncoeur, A.B. Langdon, and N.T. Gladd, "An object-oriented electromagnetic PIC code," Comput. Phys. Commun., vol. 87, pp. 199-211, 1995.

[29] E. Balagurusamy, Object Oriented Programming With $\mathrm{C}++$, New Delhi: Tata McGraw-Hill, 2008.

[30] [Online]. Available: http://ptsg.egr.msu.edu/

[31] S.C. Chapra and R.P. Canale, Numerical Methods For Engineers, New York: McGrawHill, 2015.

[32] N.A. Krall and A.W. Trivelpiece, Principles of Plasma Physics, New York: McGraw-Hill, 1973.

[33] R. Kumar, S.V. Kulkarni, and D. Bora, "Cylindrical stationary striations in surface wave produced plasma columns of argon," Phys. Plasmas, vol. 14, pp. 122101 (1-8), 2007.

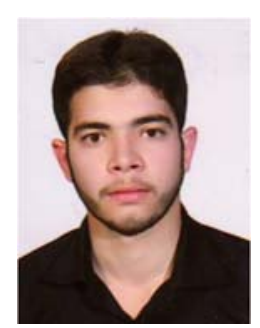

Mohammad Sadegh Soltani Gishini, born in 1988, received his Master in applied physics from Shahid Bahonar university of Kerman, Iran in 2013. He is currently working toward the doctorate degree at the Graduate University of Advanced Technology, Kerman, Iran. His research interests are applications of plasma physics and numerical modeling of gaseous discharges.

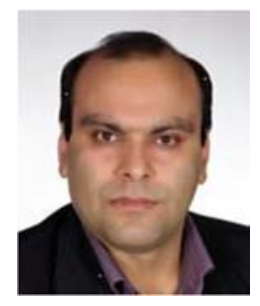

Alireza Ganjovi received his Ph.D. degree in the field of Gaseous Discharges in Electrical Engineering Department at the Indian Institute of Technology (IIT), Kanpur, India, 2011. He is currently Faculty Member of Photonics Research Institute in the Graduate University of 
Advanced Technology, Kerman, Iran. His research interests are plasma engineering and gaseous discharges and their technological applications.
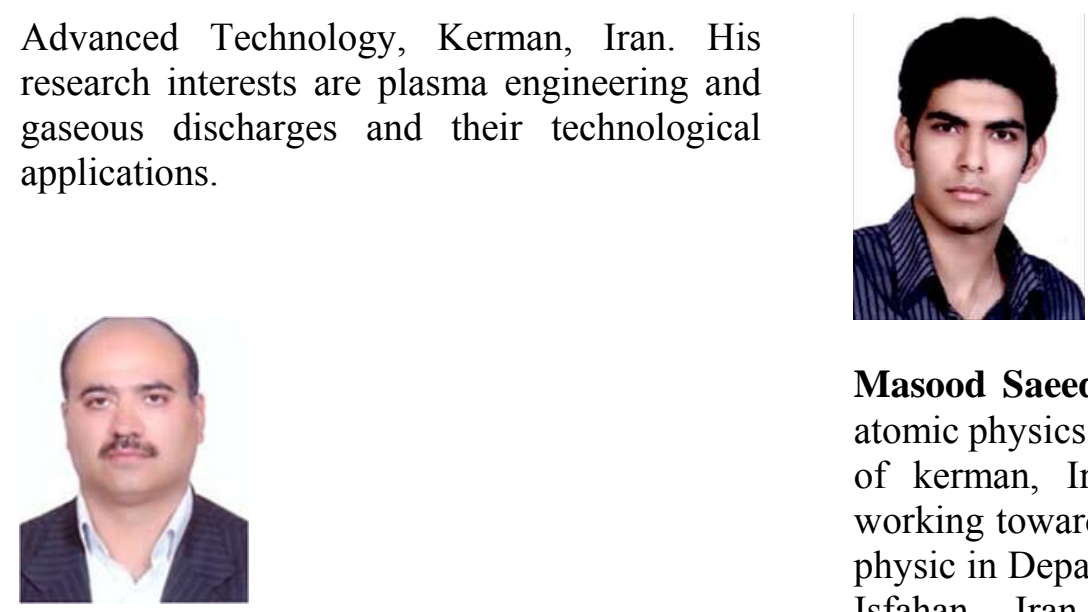

Masood Saeed received his master degree in atomic physics from Shahid Bahonar university of kerman, Iran in 2014. He is currently working toward the doctorate degree at atomic physic in Department of Physics, University of Isfahan, Iran. His research interests are computational physics, applied

Majid Taraz received his Ph.D. degree in the field of Laser physics at the Institute of Physics Electromagnetics and nonlinear optics. and Technology, Moscow, Russia, 2004. He is currently Faculty Member of Physics Faculty at Shahid Bahonar University of Kerman, Iran. His research interests are plasmas, atomic physics and their technological applications. 